\title{
Ab initio elastic tensor of cubic Ti0.5Al0.5N alloys: Dependence of elastic constants on size and shape of the supercell model and their convergence
}

Ferenc Tasnadi, Magnus Odén and Igor Abrikosov

\author{
Linköping University Post Print
}

N.B.: When citing this work, cite the original article.

Original Publication:

Ferenc Tasnadi, Magnus Odén and Igor Abrikosov, Ab initio elastic tensor of cubic Ti0.5Al0.5N alloys: Dependence of elastic constants on size and shape of the supercell model and their convergence, 2012, Physical Review B. Condensed Matter and Materials Physics, (85), 14, 144112.

http://dx.doi.org/10.1103/PhysRevB.85.144112

Copyright: American Physical Society

http://www.aps.org/

Postprint available at: Linköping University Electronic Press

http://urn.kb.se/resolve?urn=urn:nbn:se:liu:diva-77536 


\title{
$A b$ initio elastic tensor of cubic $\mathrm{Ti}_{0.5} \mathrm{Al}_{0.5} \mathrm{~N}$ alloys: Dependence of elastic constants on size and shape of the supercell model and their convergence
}

\author{
Ferenc Tasnádi, ${ }^{*}$ M. Odén, and Igor A. Abrikosov \\ Department of Physics, Chemistry and Biology (IFM), Linköping University, SE-581 83 Linköping, Sweden
}

(Received 3 November 2011; revised manuscript received 13 March 2012; published 20 April 2012)

\begin{abstract}
In this study we discuss the performance of the special quasirandom structure (SQS) method in predicting the elastic properties of $\mathrm{B} 1$ (rocksalt) $\mathrm{Ti}_{0.5} \mathrm{Al}_{0.5} \mathrm{~N}$ alloy. We use a symmetry-based projection technique, which gives the closest cubic approximate of the elastic tensor and allows us to align the SQSs of different shapes and sizes for a comparison in modeling elastic tensors. We show that the derived closest cubic approximate of the elastic tensor converges faster with respect to SQS size than the elastic tensor itself. That establishes a less demanding computational strategy to achieve convergence for the elastic constants. We determine the cubic elastic constants $\left(C_{i j}\right)$ and Zener's type elastic anisotropy $(A)$ of $\mathrm{Ti}_{0.5} \mathrm{Al}_{0.5} \mathrm{~N}$. Optimal supercells, which capture accurately both the configurational disorder and cubic symmetry of elastic tensor, result in $C_{11}=447 \mathrm{GPa}, C_{12}=158 \mathrm{GPa}$, and $C_{44}=203 \mathrm{GPa}$ with $3 \%$ of error and $A=1.40$ with $6 \%$ of error. In addition, we establish the general importance of selecting proper SQS with symmetry arguments to reliably model elasticity of alloys. We suggest the calculation of nine elastic tensor elements: $C_{11}, C_{22}, C_{33}, C_{12}, C_{13}, C_{23}, C_{44}, C_{55}$, and $C_{66}$, to analyze the performance of SQSs and predict elastic constants of cubic alloys. The described methodology is general enough to be extended for alloys with other symmetry at arbitrary composition.
\end{abstract}

DOI: 10.1103/PhysRevB.85.144112

PACS number(s): 62.20.de, 61.43.Bn

\section{INTRODUCTION}

TiAlN coatings with their good oxidation resistance and excellent mechanical properties have attracted high technological and academic interest. ${ }^{1}$ Several studies have been devoted to extend our understanding in maximizing functionality and operational efficiency of these alloys. The thermodynamics, phase stability, and spinodal decomposition in TiAlN have been analyzed, ${ }^{2,3}$ as well as the influence of nitrogen offstoichiometry ${ }^{4}$ and pressure..$^{5}$ Theoretical analysis of alloying TiAlN with Cr has resulted in a general design route to improve the thermal stability of hard coatings. ${ }^{6}$ The importance of the significant elastic anisotropy in TiAlN on the isostructural spinodal decomposition has been discussed. ${ }^{7,8}$ Though the available theoretical tools and modern supercomputers allow us to tackle complex physical phenomena in alloys, ${ }^{9}$ the prediction of anisotropic tensorial properties of substitutional alloys, like their elasticity and piezoelectricity, from first principles remains a challenging and highly requested task in computational materials science. ${ }^{10,11}$

Although ordinary scalar cluster expansion ${ }^{12}$ offers an exact treatment of the thermodynamics of alloys with its tensorial generalization ${ }^{11}$ giving an elegant description of anisotropic tensorial materials quantities of alloys, in practice the computationally less demanding special quasirandom structure (SQS) approach ${ }^{13}$ is often used instead. For example the giant piezoelectric response of ScAlN alloys ${ }^{14,15}$ or the mechanical properties of $\mathrm{TiAlN}^{7}$ have been successfully described with this approach. Using different superstructures, Mayrhofer et al. have discussed the impact of the alloy configuration on the structural, elastic properties, and phase stability in TiAlN. ${ }^{16}$ In B-doped wurtzite AlN significant configurational dependence of the piezoelectric constant has been predicted ${ }^{17}$ and electronic properties and nonlinear macroscopic polarization in III-V nitride alloys with wurtzite symmetry have been discussed. ${ }^{18,19}$
In these studies the success of the SQS approach in describing the energetics of alloys was presumed for predicting tensorial materials properties. Most of the previous theoretical works assumed the experimentally observed symmetry for the SQS supercells and focused only on the corresponding principal symmetry nonequivalent tensor elements. The SQS in principle breaks the point group symmetry of the original alloy. Thus, improperly chosen SQS supercells may result in a large discrepancy between theory and experiment or in erroneous theoretical findings.

The SQS approach does not aim to generate structures that preserve the point group symmetry of an alloy and thus to provide the proper description of tensorial properties of the alloy. In fact, different SQS supercells break the symmetry differently. The comparison of the SQSs in terms of modeling the elasticity of cubic $\mathrm{Ti}_{0.5} \mathrm{Al}_{0.5} \mathrm{~N}$ is a rather complex task. Detailed systematic studies of the application of SQS method in predicting elastic constants of alloys are required. For example, von Pezold et al. ${ }^{20}$ recently evaluated the performance of symmetrically shaped $(A \times A \times A)$ supercells for the description of elasticity in substitutional AlTi alloys. Convergence and error bars were obtained for the cubicaveraged principle cubic elastic constants $\bar{C}_{11}, \bar{C}_{12}$, and $\bar{C}_{44}$ within the supercell configuration space. However, a general concept of predicting tensorial materials properties with SQS technique is still lacking.

Here, we present a projection approach for $a b$ initio calculation of elastic constants using the SQS method to study the elastic properties of $\mathrm{B} 1 \mathrm{Ti}_{0.5} \mathrm{Al}_{0.5} \mathrm{~N}$. The idea is to search for SQS that results in an elastic tensor closest to the true one while including only the nonvanishing elements. Our results establish the importance of selecting proper supercells with symmetry arguments to reliably model elasticity of alloys. We show that supercells even with optimal short-range order (SRO) parameters may result in large noncubic elastic constants. Furthermore, we reveal that the derived closest 
cubic approximate of the elastic tensor converges faster with respect to SQS size than the elastic tensor itself. In summary, to evaluate the performance of SQS and predict the elastic properties of cubic alloys we suggest the calculation of nine elastic tensor elements, $C_{11}, C_{22}, C_{33}, C_{12}, C_{13}, C_{23}, C_{44}, C_{55}$, and $C_{66}$, instead of three, as would be the case when cubic symmetry of the supercell is assumed or 21 , as would be required in a general case.

\section{METHOD}

The techniques we applied to calculate and analyze the SQS modeled elastic constants of cubic $\mathrm{B} 1 \mathrm{Ti}_{0.5} \mathrm{Al}_{0.5} \mathrm{~N}$ alloy are described. We introduce a projection technique that allows the comparison of the elastic tensors obtained with SQS structures of different shapes and sizes. The described methodology is general enough to be extended for substitutional alloys with any symmetry and arbitrary composition.

\section{A. Special quasirandom structure approach and symmetry consideration}

The special quasirandom structure (SQS) approach ${ }^{13}$ greatly reduces the computational difficulties of modeling thermodynamic properties of random alloys. The basic structural element of the SQS model is a supercell, which is aimed to capture the structural short-range order (SRO). ${ }^{18}$ The degree of SRO is usually measured by the Warren-Cowley parameter $\alpha .{ }^{21}$ For a pseudobinary $\mathrm{A}_{1-x} \mathrm{~B}_{x} \mathrm{~N}$ alloy it is defined as $\alpha=1-P_{B}(R) / x_{B}$, where $P_{B}(R)$ is the probability of finding a $B$ atom at a distance $R$ from an $A$ atom and $x_{B}$ stands for the concentration of $B$. A perfectly random alloy is characterized by vanishing $\alpha$ s, while $\alpha>0$ and $\alpha<0$ define clustering and ordering, respectively. In principle, approximate SQS supercells with small or vanishing SROs can only be compared if the interaction parameters are known. ${ }^{9}$ In this work the atomic configurations of the supercells were obtained by including the Warren-Cowley SRO parameters of the first seven nearest-neighboring shells on the metal sublattice. A Metropolis-type simulated annealing algorithm ${ }^{22}$ was applied to achieve the closest possible model of the perfectly random alloy in all chosen size and shape models, $(A \times B \times C)$.

The SQS approach in general breaks the point group symmetry at different stages. The configurational disorder changes the microscopic local environments, which generally results in a triclinic lattice for a finite supercell. Moreover, the SQS approach allows one to use an arbitrary supercell shape and size $(A \times B \times C)$ in terms of lattice vectors. Although this arbitrariness increases the variational freedom to obtain closely vanishing SRO parameters, it also decreases the symmetry of the model. For $\mathrm{B} 1 \mathrm{Ti}_{0.5} \mathrm{Al}_{0.5} \mathrm{~N}$ alloy, the $\mathrm{SQS}$ approach results in elastic tensors with 21 nonvanishing elements. In general, the elastic tensor in the SQS approach has a symmetry class that is lower than what the alloy shows experimentally. As different SQS supercells break the symmetry somewhat differently, the comparison of the results can only be done after alignment. On the example of $\mathrm{B} 1 \mathrm{Ti}_{0.5} \mathrm{Al}_{0.5} \mathrm{~N}$ alloy we show that a projection technique can provide such an alignment.

\section{B. Calculational technique to obtain the elastic tensors}

To obtain total energies and extract the elastic constants of the supercells, density functional theory (DFT) calculations were performed using the plane-wave ultrasoft pseudopotential-based ${ }^{23}$ QUANTUM ESPRESSO program package. ${ }^{24}$ The exchange correlation energy was approximated by the Perdew-Burke-Ernzerhof generalized gradient functional (PBE-GGA). ${ }^{25}$ The plane-wave cutoff energy together with the Monkhorst-Pack sampling ${ }^{26}$ of the Brillouin zone were tested and sufficient convergence was achieved. The pseudopotentials were downloaded from the library linked to QUANTUM ESPRESSO and tested by calculating the elastic constants of bulk B1 AlN and TiN, which found to be in agreement with literature data. ${ }^{16,27}$ In obtaining the groundstate structure of SQS supercells, both the lattice parameters and the internal atomic coordinates were relaxed by using the extended molecular dynamics method with variable cell shape. ${ }^{28}$ Thus, we avoided all residual stresses, which is essential in performing an accurate comparison of the calculated

TABLE I. The Warren-Cowley pair short-range order parameters (SROs) up to the seventh neighboring shell for each SQS supercell considered in this work.

\begin{tabular}{|c|c|c|c|c|c|c|c|c|}
\hline Structure/shell & Number of atoms & 1 & 2 & 3 & 4 & 5 & 6 & 7 \\
\hline $\mathrm{L} 1_{0}{ }^{\mathrm{a}}$ & 8 & -1.0 & -1.0 & -1.0 & -1.0 & -1.0 & -1.0 & -1.0 \\
\hline$(2 \times 2 \times 2)$ & 16 & -0.16 & 0.0 & -0.16 & 1.0 & -0.16 & 0.0 & -0.16 \\
\hline$(2 \times 3 \times 2)$ & 24 & -0.11 & 0.0 & -0.08 & 0.33 & -0.06 & -0.08 & 0.03 \\
\hline$(4 \times 3 \times 2)$ & 48 & 0.0 & 0.0 & 0.0 & 0.0 & -0.06 & 0.0 & 0.0 \\
\hline$(4 \times 3 \times 2)^{* b}$ & 48 & -0.14 & 0.28 & -0.10 & 0.14 & 0.0 & -0.17 & -0.01 \\
\hline $\mathrm{C} 1-(2 \times 2 \times 2)^{\mathrm{a}, \mathrm{c}}$ & 64 & -0.33 & 1.0 & -0.33 & 1.0 & -0.33 & 1.0 & -0.33 \\
\hline $\mathrm{C} 3-(2 \times 2 \times 2)^{\mathrm{a}, \mathrm{c}}$ & 64 & 0.0 & -1.0 & 0.0 & -1.0 & 0.0 & -1.0 & 0.0 \\
\hline $\mathrm{B} 1-(2 \times 2 \times 2)^{\mathrm{a}, \mathrm{d}}$ & 64 & 0.0 & 0.0 & 0.0 & -0.33 & 0.0 & 0.0 & 0.0 \\
\hline$(4 \times 3 \times 4)$ & 96 & 0.0 & 0.0 & 0.0 & 0.0 & 0.0 & 0.0 & 0.0 \\
\hline$(4 \times 4 \times 3)$ & 96 & 0.0 & 0.0 & -0.01 & -0.01 & -0.01 & 0.0 & 0.0 \\
\hline$(4 \times 4 \times 4)$ & 128 & 0.0 & 0.0 & 0.0 & 0.0 & 0.0 & 0.0 & 0.0 \\
\hline
\end{tabular}

\footnotetext{
${ }^{\mathrm{a}}$ The structure is based on the fcc Bravais cell.

${ }^{\mathrm{b}}$ The * marks a different atomic configuration in the supercell.

${ }^{\mathrm{c}}$ The supercell was obtained by Mayrhofer et al. in Ref. 16.

${ }^{\mathrm{d}}$ The supercell was obtained by von Pezold et al. in Ref. 20.
} 
elastic tensors. In this dynamics, a value of $0.02 \mathrm{kbar}$ was taken as convergence threshold for the pressure. The elastic constants were calculated via the second-order Taylor expansion of the total energy

$$
C_{i j}=\left.\frac{1}{V_{0}} \frac{\partial^{2} E\left(\epsilon_{1}, \ldots, \epsilon_{6}\right)}{\partial \epsilon_{i} \partial \epsilon_{j}}\right|_{0},
$$

where Voigt's notation is used to describe the strain $\epsilon$ and elastic tensor $C_{i j} .{ }^{29,30}$ To obtain the entire elastic tensor, 21 different distortions were applied without volume conservation. The elastic constants were calculated by standard finite difference technique from total energy data obtained with \pm $1 \%$ and $\pm 2 \%$ distortions.

\section{Projection of the elastic tensor to the closest elastic tensor of higher symmetry}

In this section we describe the projection technique introduced by Moakher et al. ${ }^{31}$ to derive the closest elastic tensors with cubic symmetry in the modeling of $\mathrm{Ti}_{0.5} \mathrm{Al}_{0.5} \mathrm{~N}$

$$
\begin{aligned}
P^{\text {cub }} & =\left(\begin{array}{lcc}
p^{\text {cub }} & 0_{9 \times 12} \\
0_{12 \times 9} & 0_{12 \times 12}
\end{array}\right), \\
p^{\text {cub }}= & \left(\begin{array}{lcc}
1 / 3 & 1 / 3 & 1 / 3 \\
1 / 3 & 1 / 3 & 1 / 3 \\
1 / 3 & 1 / 3 & 1 / 3 \\
0 & 0 & 0 \\
0 & 0 & 0 \\
0 & 0 & 0 \\
0 & 0 & 0 \\
0 & 0 & 0 \\
0 & 0 & 0
\end{array}\right.
\end{aligned}
$$

$\left.\begin{array}{cccccc}0 & 0 & 0 & 0 & 0 & 0 \\ 0 & 0 & 0 & 0 & 0 & 0 \\ 0 & 0 & 0 & 0 & 0 & 0 \\ 1 / 3 & 1 / 3 & 1 / 3 & 0 & 0 & 0 \\ 1 / 3 & 1 / 3 & 1 / 3 & 0 & 0 & 0 \\ 1 / 3 & 1 / 3 & 1 / 3 & 0 & 0 & 0 \\ 0 & 0 & 0 & 1 / 3 & 1 / 3 & 1 / 3 \\ 0 & 0 & 0 & 1 / 3 & 1 / 3 & 1 / 3 \\ 0 & 0 & 0 & 1 / 3 & 1 / 3 & 1 / 3\end{array}\right)$.

by supercell method. The 21 inequivalent elastic constants of a triclinic system can be given as a vector with 21 components

$$
\begin{aligned}
C= & \left(C_{11}, C_{22}, C_{33}, \sqrt{2} C_{23}, \sqrt{2} C_{13}, \sqrt{2} C_{12}, 2 C_{44}, 2 C_{55},\right. \\
& 2 C_{66}, 2 C_{14}, 2 C_{25}, 2 C_{36}, 2 C_{34}, 2 C_{15}, 2 C_{26}, 2 C_{24}, \\
& \left.2 C_{35}, 2 C_{16}, 2 \sqrt{2} C_{56}, 2 \sqrt{2} C_{46}, 2 \sqrt{2} C_{45}\right),
\end{aligned}
$$

where the normalization factors $(2, \sqrt{2})$ ensure the invariance of the Euclidean norm on the representation, whether it is vector or matrix. For the corresponding basis vectors see Ref. 32. In general, projector $P^{\text {sym }}$ gives the closest elastic tensor with higher point group symmetry $C^{\text {sym }}$ as

$$
C^{\text {sym }}=P^{\text {sym }} C \text {. }
$$

The term closest is used here in the sense that the Euclidean distance $\left\|C-C^{\text {sym }}\right\|$ is minimal. In modeling $\mathrm{B} 1 \mathrm{Ti}_{0.5} \mathrm{Al}_{0.5} \mathrm{~N}$ alloy one should apply the projector given as a $21 \times 21$ matrix, ${ }^{31,32}$
Then the obtained closest cubic elastic tensor is written as

$$
\begin{aligned}
C^{\mathrm{cub}}= & \left(\bar{C}_{11}, \bar{C}_{11}, \bar{C}_{11}, \sqrt{2} \bar{C}_{12}, \sqrt{2} \bar{C}_{12}, \sqrt{2} \bar{C}_{12}, 2 \bar{C}_{44},\right. \\
& \left.2 \bar{C}_{44}, 2 \bar{C}_{44}, 0,0,0,0,0,0,0,0,0,0,0,0\right),
\end{aligned}
$$

where the projected cubic elastic constants $\bar{C}_{11}, \bar{C}_{12}$, and $\bar{C}_{44}$ are calculated via simple averaging,

$$
\begin{aligned}
& \bar{C}_{11}=\frac{C_{11}+C_{22}+C_{33}}{3} \quad \bar{C}_{12}=\frac{C_{12}+C_{13}+C_{23}}{3} \\
& \bar{C}_{44}=\frac{C_{44}+C_{55}+C_{66}}{3} .
\end{aligned}
$$

We may also call them cubic-averaged elastic constants, as Eq. (6) is equivalent with averaging over the three crystallographic directions, [100], [010], and [001]. This directional averaging was also used by von Pezold et al. ${ }^{20}$ in searching for optimized supercells in AlTi alloys. According to Eq. (6) one needs the nine independent tensor elements $C_{11}, C_{22}, C_{33}, C_{23}, C_{13}, C_{12}, C_{44}, C_{55}$, and $C_{66}$ to obtain the closest cubic projection of an elastic tensor with arbitrary symmetry. In case of true cubic point symmetry Eq. (6) results in the well-known cubic identities of the elastic constants. The rigid mathematical derivation of the projectors for all symmetry classes-monoclinic, orthorhombic, tetragonal, trigonal, hexagonal, cubic, and isotropic-can be found in Refs. 31 and 32 . We explicitly present the projector for hexagonal point group symmetry in the supplemental material. ${ }^{33}$

\section{RESULTS AND DISCUSSION}

In this section we present and compare the elastic tensors obtained for the cubic (B1) $\mathrm{TiA}_{0.5} \mathrm{l}_{0.5} \mathrm{~N}$ alloy. The SQS supercells with shape and size, $(2 \times 2 \times 2),(2 \times 3 \times 2)$, $(4 \times 3 \times 2),(4 \times 3 \times 4),(4 \times 4 \times 3)$, and $(4 \times 4 \times 4)$ were generated as described previously. The SQS supercell sizes are measured in terms of the face centered cubic (fcc) unit vectors. The obtained internal atomic configurations of the supercells are given in the supplemental material. ${ }^{33}$ We also present results for the ordered $\mathrm{L}_{0}$ structure and three other structures, denoted here by $\mathrm{C} 1-(2 \times 2 \times 2)$, C3- $(2 \times 2 \times 2)$, and B1$(2 \times 2 \times 2)$. The latter structures are based on the fcc Bravais cell. The C1- $(2 \times 2 \times 2)$ and C3- $(2 \times 2 \times 2)$ were created by Mayrhofer et al. ${ }^{16}$ considering the number of bonds between the host and doping atoms. The C3- $(2 \times 2 \times 2)$ structure was 


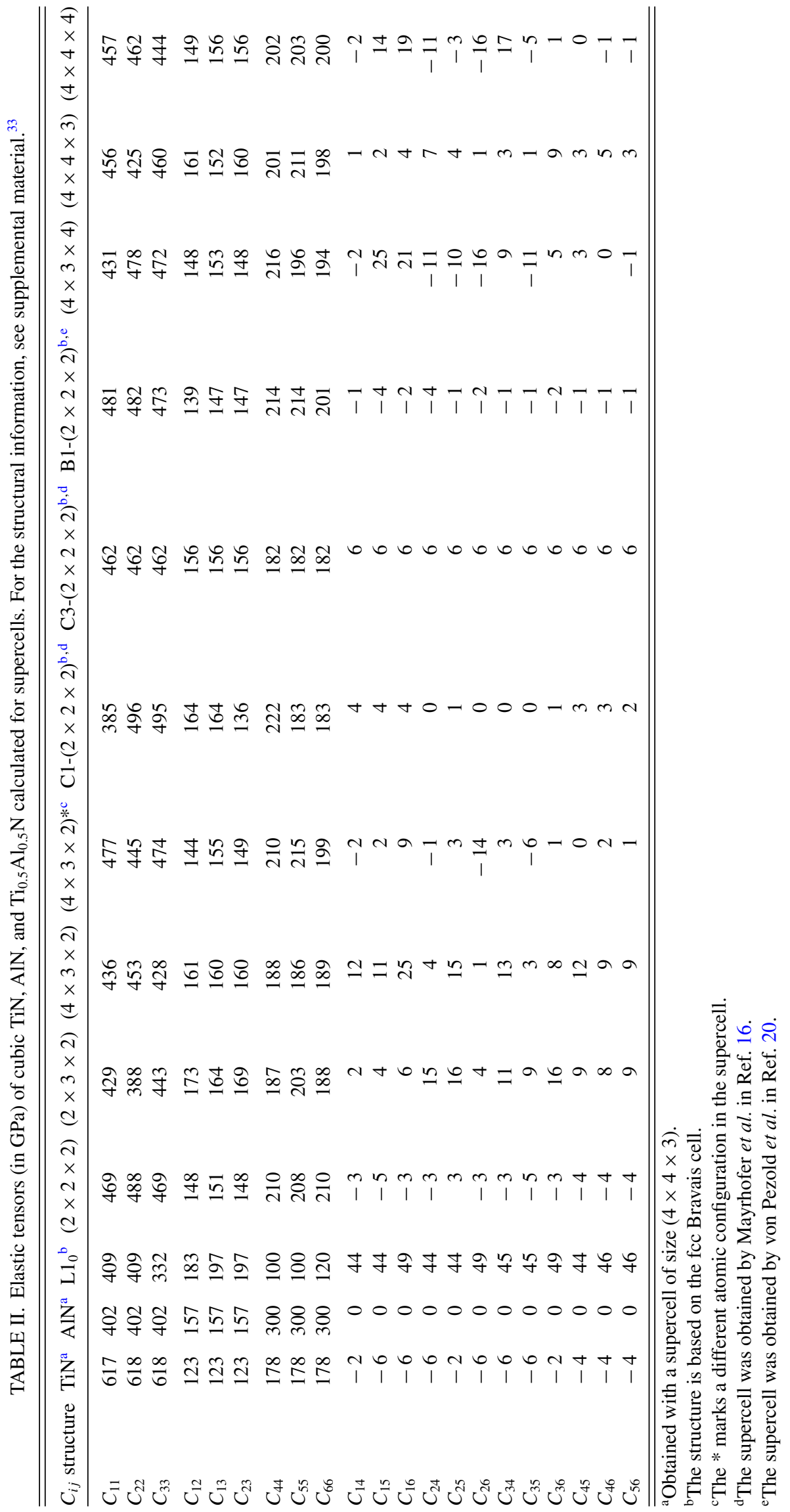


TABLE III. The projected principal cubic elastic constants and the derived Zener's elastic anisotropy $\bar{A}=2 \bar{C}_{44} /\left(\bar{C}_{11}-\bar{C}_{12}\right)$ of $\mathrm{Ti}_{0.5} \mathrm{Al}_{0.5} \mathrm{~N}$ obtained with the different structural models. The bold written values are our most accurate results.

\begin{tabular}{lllll}
\hline \hline Structure/constant & $\bar{C}_{11}$ & $\bar{C}_{12}$ & $\bar{C}_{44}$ & $\bar{A}$ \\
\hline $\mathrm{L} 1_{0}{ }^{\mathrm{a}}$ & 384 & 193 & 107 & 1.12 \\
$(2 \times 2 \times 2)$ & 475 & 149 & 209 & 1.28 \\
$(2 \times 3 \times 2)$ & 420 & 169 & 193 & 1.53 \\
$(4 \times 3 \times 2)$ & 439 & 160 & 188 & 1.35 \\
$(4 \times 3 \times 2)^{*}, \mathrm{~b}$ & 465 & 149 & 208 & 1.32 \\
$\mathrm{C} 1-(2 \times 2 \times 2)^{\mathrm{a}, \mathrm{c}}$ & 459 & 155 & 196 & 1.29 \\
$\mathrm{C} 3-(2 \times 2 \times 2)^{\mathrm{a}, \mathrm{c}}$ & 462 & 156 & 182 & 1.19 \\
$\mathrm{~B} 1-(2 \times 2 \times 2)^{\mathrm{a}, \mathrm{d}}$ & 479 & 144 & 215 & 1.29 \\
$(4 \times 3 \times 4)$ & 460 & 150 & 202 & 1.30 \\
$(\mathbf{4} \times \mathbf{4} \times \mathbf{3})$ & $\mathbf{4 4 7}$ & $\mathbf{1 5 8}$ & $\mathbf{2 0 3}$ & $\mathbf{1 . 4 0}$ \\
$(4 \times 4 \times 4)$ & 454 & 154 & 202 & 1.34 \\
\hline
\end{tabular}

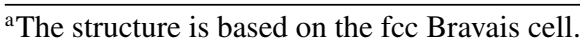

${ }^{\mathrm{b}}$ The * marks a different atomic configuration in the supercell.

${ }^{\mathrm{c}}$ The supercell was obtained by Mayrhofer et al. in Ref. 16.

${ }^{\mathrm{d}}$ The supercell was obtained by von Pezold et al. in Ref. 20 .

designed to preserve the cubic symmetry. The B1- $(2 \times 2 \times 2)$ structure has been obtained by von Pezold using a Monte Carlo scheme and averaging over the three orthogonal main crystallographic directions. ${ }^{20}$ The SRO parameters of all structures are summarized in Table I. The star $(*)$ marks the SQS structure that is less random. See supplemental material ${ }^{33}$ for a detailed structural comparison of the supercells. We note that the larger structural deviations in the case of $(4 \times 3 \times 4)$ already indicate deviating behavior for the analysis of elastic constants.

For each of these structures the full elastic tensors have been calculated. They are summarized in Table II. All the obtained tensors exhibit deviations from a strict cubic symmetry. Table II also lists the elastic tensors of B1 TiN and AlN obtained with the $(4 \times 4 \times 3)$ supercell. Here one should find the cubic symmetry of elastic constants, therefore deviations give an estimate of the numerical error in the usual firstprinciples calculation of the elastic constants. We note also that the elastic constants of B1 TiN and AlN show good agreement with the literature data. ${ }^{16,27}$ As the $\mathrm{C} 3-(2 \times 2 \times 2)$ supercell preserves the cubic symmetry, its elastic tensor fulfills the cubic relationships. The other nonvanishing elements define our numerical accuracy, which is estimated to be around $3 \%=(6 / 463+6 / 182+6 / 156) / 3 \%$. Since one gets the same $3 \%$ numerical error in the case of bulk B1 TiN and a negligible one for $\mathrm{B} 1 \mathrm{AlN}$, we assume that $3 \%$ is the characteristic numerical error through the following analysis. One sees from the data in Table II that some of the SQSs result in large noncubic elements. The $(4 \times 3 \times 4) \mathrm{SQS}$, for example, gives relatively large values for $C_{16}, C_{15}$, and $C_{25}$, which then contribute to the value of $\|C\|$ in accordance to Eq. (2).

Thereafter, we extract the closest cubic elastic tensors by using the cubic projector from Eq. (4). The obtained projected cubic elastic constants are summarized in Table III. In Fig. 1 we present an important characteristics of the cubic-averaged elastic constants $\bar{C}_{11}, \bar{C}_{12}$, and $\bar{C}_{44}$. The figure shows the nine calculated elastic constants together with the corresponding
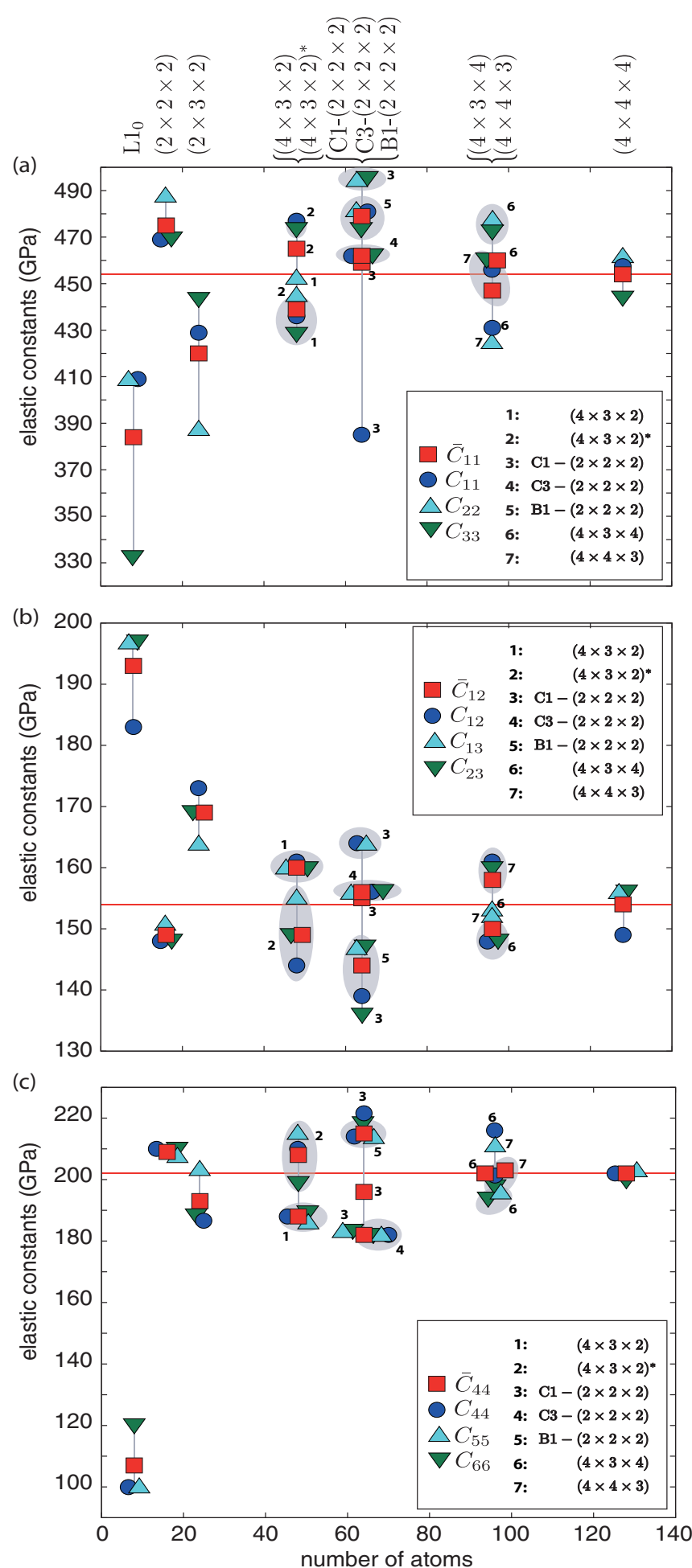

FIG. 1. (Color online) The calculated nine projected cubic elastic constants of $\mathrm{Ti}_{0.5} \mathrm{Al}_{0.5} \mathrm{~N}$ together with the derived cubic-averaged elastic constants.

averaged values for each supercell. It presents clearly that the cubic-averaged elastic constants converge faster with respect to the number of atoms. Accordingly, this nine-dimensional analysis provides a less demanding technique to achieve convergence for the elastic constants.

To give more insight into the accuracy of SQS performance, we calculate the distance variations $\left\|C-C^{\text {cub }}\right\| /\|C\|$. 


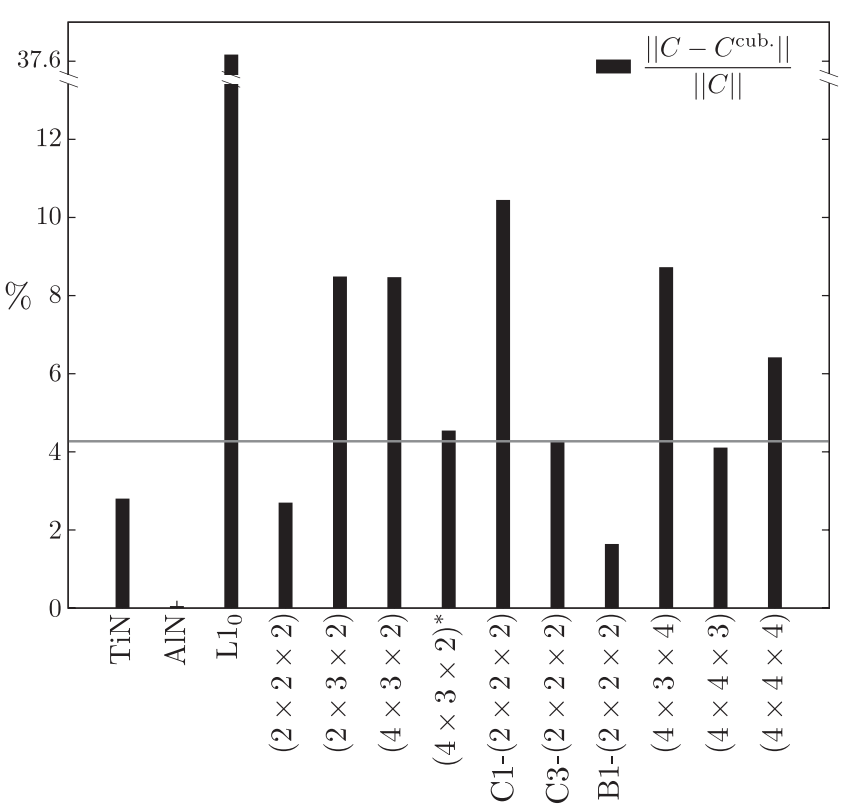

FIG. 2. (Color online) Calculated euclidian distance deviations $\left\|C-C^{\mathrm{cub}}\right\| /\|C\|$ obtained in the 21-dimensional space, see Eqs. (2) and (5).

The obtained deviations are shown in Fig. 2. Since the C3- $(2 \times 2 \times 2)$ supercell should have cubic symmetry, its $\left\|C-C^{\mathrm{cub}}\right\| /\|C\|$ value defines the numerical threshold in Fig. 2. It is found to be around $4.3 \%$. According to Fig. 2, only the $(2 \times 2 \times 2)$, C $3-(2 \times 2 \times 2), \mathrm{B} 1-(2 \times 2 \times 2)$, and $(4 \times 4 \times 3)$ supercells give cubic elastic tensors within the most general 21 -dimensional vector space. The $(4 \times 3 \times 2)^{*}$ and $(4 \times 4 \times 4)$ are the candidates that closely exhibit a cubic symmetry of the elastic tensors. The ordered $\mathrm{L}_{0}$ structure results in the largest deviation from cubic symmetry. While the $(2 \times 2 \times 2)$ supercell with relative large SRO parameters fulfills the cubic symmetry requirement, the larger and perfectly random $(4 \times 3 \times 4)$ supercell does not. This underlines the importance of designing SQS supercells with the support of the point group symmetry in dealing with tensorial properties of alloys. From Fig. 2 and the SRO parameters we conclude that among the tested structures our $(4 \times 4 \times 3)$ model should be taken as the best model to study the elasticity in cubic $\mathrm{Ti}_{0.5} \mathrm{Al}_{0.5} \mathrm{~N}$. Accordingly, cubic $\mathrm{B} 1 \mathrm{Ti}_{0.5} \mathrm{Al}_{0.5} \mathrm{~N}$ alloy has the elastic constant of $C_{11}=447 \mathrm{GPa}, C_{12}=158 \mathrm{GPa}$, and $C_{44}=203 \mathrm{GPa}$ within $3 \%$ of numerical error. Note that the elastic constants calculated by us for the largest $(4 \times 4 \times 4)$ supercell using the projection technique are also within this $3 \%$, and therefore the results are converged (see Fig. 4). We also see that when using ad hoc or inadequate structures, such as the $\mathrm{L} 1_{0}$, one ends up with large $22-50 \%$ errors. It is worth mentioning that in the case of the $(4 \times 3 \times 2)$ and $(4 \times 3 \times 4)$ SQSs the large noncubic elastic constants can be related to the large deviations in Fig. 2.

The projection technique allows us to evaluate the supercells in a smaller, only nine-dimensional vector space by omitting the principal noncubic tensor elements. We deal only with the following nine elastic constants: $C_{11}, C_{22}, C_{33}, C_{12}$, $C_{13}, C_{23}, C_{44}, C_{55}$, and $C_{66}$. The deviations of these constants from the projected cubic elastic constants $\bar{C}_{11}, \bar{C}_{12}$, and $\bar{C}_{44}$
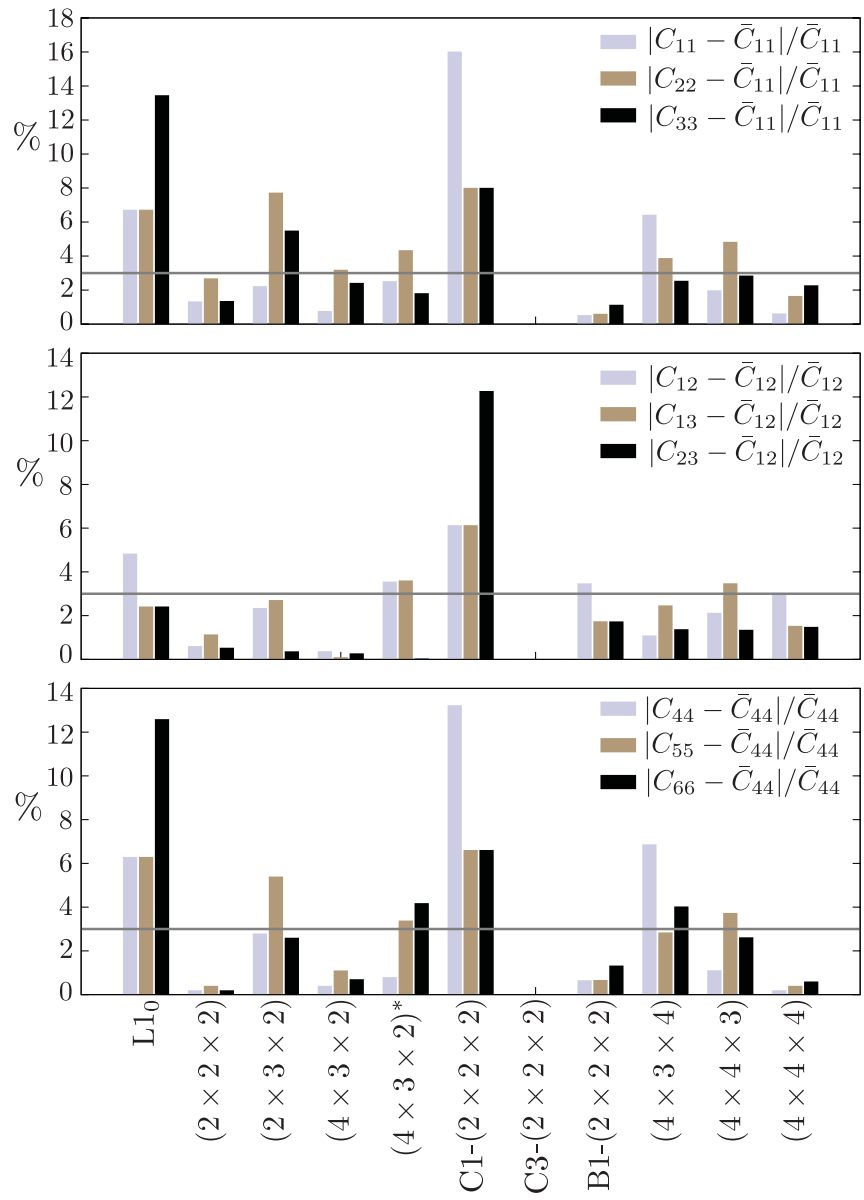

FIG. 3. (Color online) Comparison of the calculated elastic tensor elements with the projected principal cubic elastic constants in $\mathrm{Ti}_{0.5} \mathrm{Al}_{0.5} \mathrm{~N}$.

are shown in Fig. 3. In this figure, the three columns for each supercell give the deviations along the three crystallographic directions [100], [010], and [001]. One can see in the figure, where the horizontal lines show our $3 \%$ error threshold, that only three supercells, the $(2 \times 2 \times 2)$, C $3-(2 \times 2 \times 2)$, and $(4 \times 4 \times 4)$ result in cubic elastic constants. The comparison of the SRO parameters of these structures makes $(4 \times 4 \times 4)$ ultimately the best model in this nine-dimensional analysis. Similarly in Fig. 2, the $(4 \times 4 \times 3)$ supercell performs very well, while the totally random $(4 \times 3 \times 4)$ SQS with better SRO parameters does not. This fact might be related to the observed large deviations in the relaxed lattice parameters (see supplemental material). ${ }^{33}$ As the projection technique omits the principal noncubic elastic constants, the values in Fig. 3 are free from these contributions.

In Fig. 4 the relative deviations of elastic constants are plotted with respect to the values obtained for the $(4 \times 4 \times 4)$ SQS. One can see that the $(4 \times 3 \times 4),(4 \times 4 \times 3)$, and $(4 \times 4 \times 4)$ supercells result in the same elastic constants within the $3 \%$ numerical error confirming good convergence of $\bar{C}_{i j}$ seen in Fig. 1. Furthermore, Fig. 4 can be used to distinguish the supercells that give good approximations of the cubic elastic constants in Fig. 3. For example, the $(4 \times 3 \times 2)$ structure performs well in Fig. 3 , but it results in the $\bar{C}_{i j}$ deviating more than the numerical error in Figs. 1 and 4. Thus, 


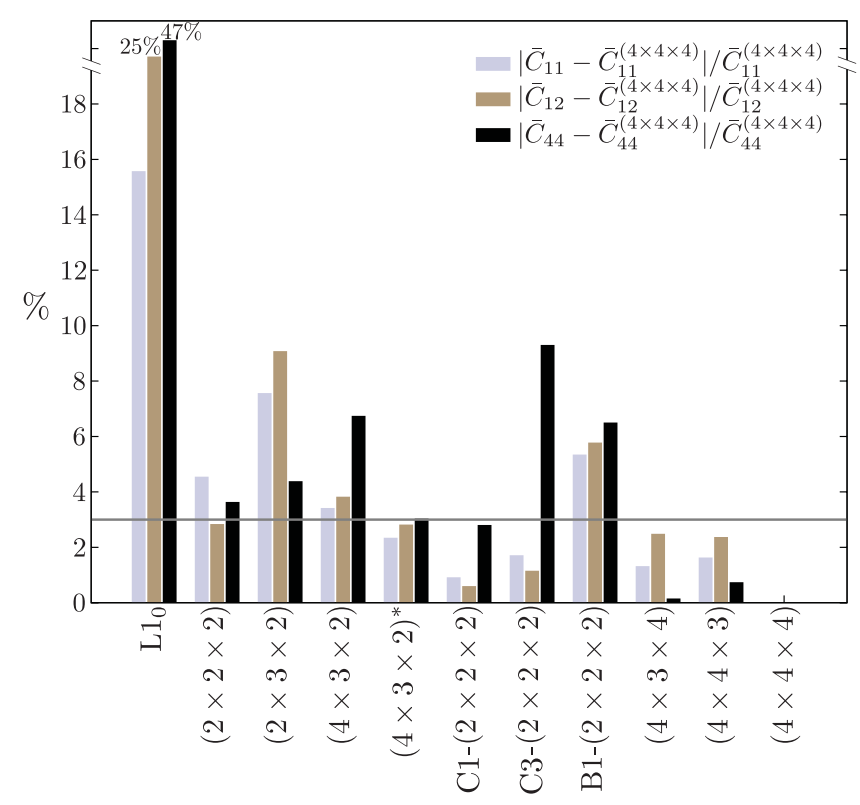

FIG. 4. (Color online) The calculated projected cubic elastic constants of $\mathrm{Ti}_{0.5} \mathrm{Al}_{0.5} \mathrm{~N}$ relative to the values obtained for the $(4 \times 4 \times 4)$ SQS model.

$(4 \times 3 \times 2)$ does not give converged $\bar{C}_{i j}$, while the $(4 \times 4 \times 3)$ SQS does. We conclude that one can similarly analyze the performance of the supercells in terms of predicting elastic constants of alloys with cubic symmetry within this smaller nine-dimensional subspace. Such analysis is highly relevant for selecting SQSs to be used in more expensive simulations [e.g., of temperature-dependent elastic constants using $a b$ initio molecular dynamics (AIMD)].

The large directional variation of the elastic constants (see the length of the vertical lines in Fig. 1), indicate a strong directional variation of the materials elastic anisotropy. Elastic anisotropy in TiAlN alloys has a huge impact on the material's mechanical properties. ${ }^{7}$ Its accurate prediction is very important. Using the projected cubic elastic constants, we derive the averaged Zener's elastic anisotropy

$$
\bar{A}=\frac{2 \bar{C}_{44}}{\bar{C}_{11}-\bar{C}_{12}} .
$$

The obtained values are listed in Table III and plotted in Fig. 5. The numerical error accumulates in this expression in the numerator. The $(4 \times 4 \times 3)$ SQS gives the value of the Zener's elastic anisotropy in $\mathrm{Ti}_{0.5} \mathrm{Al}_{0.5} \mathrm{~N}$ as $\bar{A}=1.40$ with around $6 \%$ numerical error. For the two most optimal supercells, $(4 \times$ $4 \times 3)$ and $(4 \times 4 \times 4)$, one obtains a $5 \%$ difference between the elastic anisotropy values. Figure 5 shows not only $\bar{A}$ but also its variation along the three orthogonal crystallographic directions, [100], [010], and [001]. For example, in the [100]

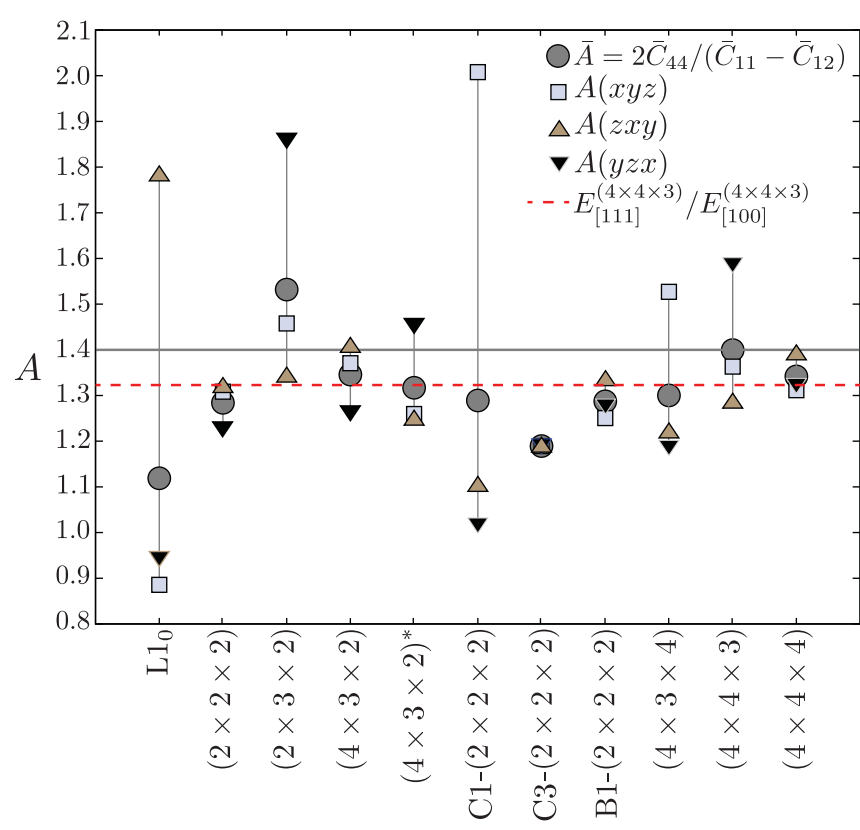

FIG. 5. (Color online) Zener's elastic anisotropy values in $\mathrm{Ti}_{0.5} \mathrm{Al}_{0.5} \mathrm{~N}$ for each structural model considered in this study. The horizontal solid line shows the value of $\bar{A}$ obtained for the $(4 \times 4 \times 3)$ SQS.

direction one has $A(x, y, z)=2 C_{44} /\left(C_{11}-C 12\right)$, in [010] $A(y z x)=2 C_{66} /\left(C_{33}-C_{13}\right)$. These orientational variations should vanish in the case of true cubic symmetry. As the figure shows one may get a large orientation dependence $[\approx 55 \%$, see $\mathrm{C} 1-(2 \times 2 \times 2)$ ] for a supercell being far from fulfilling cubic symmetry of elastic constants. The sizes of the variations correlate with the deviations in Figs. 1 and 3. However, as seen in Fig. $1, \bar{A}$ also converges faster with the size of the SQSs. Accordingly, Fig. 5 gives a similar tool to analyze the performance of the supercells in predicting the elastic constants of cubic alloys. In the Reuss averaging method, with assumed uniform stress distribution, the strain ratio $\epsilon_{[200]} / \epsilon_{[111]}=E_{[111]} / E_{[100]}$ can be used to extract elastic anisotropy from experiments. Here $E_{[h k l]}$ denotes the directional Young's elastic moduli. Using our most accurate supercell model of $(4 \times 4 \times 3)$ the strain ratio $\epsilon_{[200]} / \epsilon_{[111]}$ is 1.32 in $\mathrm{Ti}_{0.5} \mathrm{Al}_{0.5} \mathrm{~N}$ (see Fig. 5). This value deviates from $\vec{A}=1.40$ with less than $6 \%$ numerical error.

The elasticity of polycrystalline $\mathrm{Ti}_{0.5} \mathrm{Al}_{0.5} \mathrm{~N}$ is usually discussed in terms of the Reuss and Voigt bulk $\left(B_{R}, B_{V}\right)$, the shear moduli $\left(G_{R}, G_{V}\right)$, the Young's modulus $\left(E_{V / G}\right)$, and the Poisson ratio $\left(v_{V / G}\right)$ (see Ref. 33 for the expressions). The polycrystalline averaged quantities obtained for $(4 \times 4 \times 3)$ and $(4 \times 4 \times 4)$ SQSs are summarized in Table IV. One can see that the obtained values agree to each other. In particular,

TABLE IV. The polycrystalline bulk $(B)$, shear $(G)$, Young $(E)$ moduli in units of GPa and the Poisson ratio of $\mathrm{Ti}_{0.5} \mathrm{Al}_{0.5} \mathrm{~N}$ obtained with the $(4 \times 4 \times 3)$ and $(4 \times 4 \times 4)$ supercells.

\begin{tabular}{lccccccr}
\hline \hline Structure & $B_{V}$ & $B_{R}$ & $G_{V}$ & $G_{R}$ & $E_{V}$ & $E_{R}$ & $v_{V}$ \\
\hline$(4 \times 4 \times 3)$ & 254 & 254 & 180 & 174 & 437 & 425 & 0.21 \\
$(4 \times 4 \times 4)$ & 254 & 254 & 181 & 176 & 439 & 429 & 0.21 \\
\hline \hline
\end{tabular}


$B_{\mathrm{R}}=B_{\mathrm{V}}$ clearly shows fulfillment of the requirement of a cubic crystal.

\section{CONCLUSION}

In this study we discuss the performance of superstructures, including approximate special quasirandom structure (SQS) supercells in predicting the elasticity of cubic $\mathrm{B} 1 \mathrm{Ti}_{0.5} \mathrm{Al}_{0.5} \mathrm{~N}$ alloy. Though the SQS approach provides a successful scheme to model and predict the thermodynamics of alloys, the technique is not aimed directly for a description of tensorial materials properties. Thus, its straightforward application cannot provide an unambiguous description of elasticity in random alloys.

Here, we applied a symmetry-based projection technique to accurately predict the cubic elastic tensor of $\mathrm{B} 1 \mathrm{Ti}_{0.5} \mathrm{Al}_{0.5} \mathrm{~N}$ alloy within the SQS approach. We derived from $a b$ initio calculations the closest cubic elastic tensor of $\mathrm{B} 1 \mathrm{Ti}_{0.5} \mathrm{Al}_{0.5} \mathrm{~N}$ by using several supercells. With the help of these derived cubic projected elastic constants we presented a detailed comparison of the results obtained with SQSs of different shape and size. The $(4 \times 4 \times 3)$ and $(4 \times 4 \times 4)$ supercells provided us the optimal models of both configurational disorder and symmetry of elastic tensor. They resulted in $C_{11}=447 \mathrm{GPa}, C_{12}=158 \mathrm{GPa}$, and $C_{44}=203 \mathrm{GPa}$ with $3 \%$ of numerical error. Furthermore, the derived Zener's type elastic anisotropy has a value of $\bar{A}=1.40$ with $6 \%$ of error.

Through the discussion of the calculated full elastic tensors with 21 constants, we established that supercells with good SRO parameters may include large noncubic elements and that supercells with somewhat worse SRO parameters can approximate elastic tensors fairly accurately. We showed that by using only nine elements, $C_{11}, C_{22}, C_{33}, C_{12}, C_{13}, C_{23}$, $C_{44}, C_{55}$, and $C_{66}$, from the tensors, one can evaluate the SQS models in terms of predicting the elastic constants of cubic $\mathrm{Ti}_{0.5} \mathrm{Al}_{0.5} \mathrm{~N}$. Moreover, we revealed that the derived closest cubic approximation of the elastic tensor converges faster with respect to SQS size than the elastic tensor itself. This observation establishes a less demanding computational strategy to achieve convergence for the elastic constants. The obtained large directional (crystallographic) variation of the results underlined the investigation of the Zener's type elastic anisotropy. We also showed that the deviations between the three equivalent Zener-type anisotropy factors for SQSs oriented along the [100], [010], and [001] directions, might differ. This further underlined the uncertainty in the straightforward application of the SQS approach in predicting tensorial materials quantities.

In summary, we have accurately predicted the cubic elastic constants of $\mathrm{B} 1 \mathrm{Ti}_{0.5} \mathrm{Al}_{0.5} \mathrm{~N}$ alloy. We suggest the calculation of nine elastic tensor elements $-C_{11}, C_{22}, C_{33}, C_{12}, C_{13}, C_{23}$, $C_{44}, C_{55}$, and $C_{66}$ - to obtain faster convergence with respect to the SQS size for the elastic constants of cubic alloys in evaluating the performance of supercells.

\section{ACKNOWLEDGMENTS}

This work was supported by the Swedish Foundation for Strategic Research (SSF) project Designed Multicomponent coatings, MultiFilms and the Swedish Research Council (VR). Calculations were performed at the Swedish National Infrastructure for Computing (SNIC). *tasnadi@ifm.liu.se

${ }^{1}$ A. Hörling, L. Hultman, M. Odén, J. Sjölén, and L. Karlsson, Surf. Coat. Technol. 191, 384 (2002).

${ }^{2}$ B. Alling, A. V. Ruban, A. Karimi, O. E. Peil, S. I. Simak, L. Hultman, and I. A. Abrikosov, Phys. Rev. B 75, 045123 (2007).

${ }^{3}$ P. Mayrhofer, D. Musics, and J. M. Schenider, Appl. Phys. Lett. 88, 071922 (2006).

${ }^{4}$ B. Alling, A. Karimi, L. Hultman, and I. A. Abrikosov, Appl. Phys. Lett. 92, 071903 (2008).

${ }^{5}$ B. Alling, M. Odén, L. Hultman, and I. A. Abrikosov, Appl. Phys. Lett. 95, 181906 (2009).

${ }^{6}$ H. Lind, R. Forsén, B. Alling, N. Ghafoor, F. Tasnádi, M. P. Johansson, I. A. Abrikosov, and M. Odén, Appl. Phys. Lett. 99, 091903 (2011).

${ }^{7}$ F. Tasnádi, I. A. Abrikosov, L. Rogström, J. Almer, M. P. Johansson, and M. Odén, Appl. Phys. Lett. 97, 231902 (2010).

${ }^{8}$ I.A. Abrikosov, A. Knutsson, B. Alling, F. Tasnádi, H. Lind, L. Hultman, and M. Odén, Materials 4, 1599 (2011).

${ }^{9}$ A. V. Ruban and I. A. Abrikosov, Rep. Prog. Phys. 71, 046501 (2008).

${ }^{10}$ J. Z. Liu, A. van de Walle, G. Ghosh, and M. Asta, Phys. Rev. B 72, 144109 (2005).

${ }^{11}$ A. van de Walle, Nature Mater. 7, 455 (2008).

${ }^{12}$ J. M. Sanchez, Phys. Rev. B 81, 224202 (2010).

${ }^{13}$ A. Zunger, S.-H. Wei, L. G. Ferreira, and J. E. Bernard, Phys. Rev. Lett. 65, 353 (1990).
${ }^{14}$ F. Tasnádi, B. Alling, C. Höglund, G. Wingqvist, J. Birch, L. Hultman, and I. A. Abrikosov, Phys. Rev. Lett. 104, 137601 (2010).

${ }^{15}$ G. Wingqvist, F. Tasnádi, A. Zukauskaite, J. Birch, H. Arwin, and L. Hultman, Appl. Phys. Lett. 97, 112902 (2010).

${ }^{16}$ P. H. Mayrhofer, D. Music, and J. M. Schneider, J. Appl. Phys. 100, 094906 (2006).

${ }^{17}$ F. Tasnádi, I. A. Abrikosov, and I. Katardjiev, Appl. Phys. Lett. 94, 151911 (2009)

${ }^{18}$ K. A. Mäder and A. Zunger, Phys. Rev. B 51, 10462 (1995).

${ }^{19}$ F. Bernardini and V. Fiorentini, Phys. Rev. B 64, 085207 (2001).

${ }^{20}$ J. von Pezold, A. Dick, M. Friák, and J. Neugebauer, Phys. Rev. B 81, 094203 (2010).

${ }^{21}$ J. Cowley, Phys. Rev. 77, 669 (1950).

${ }^{22}$ N. Metropolis, A. W. Rosenbluth, M. N. Rosenbluth, A. H. Teller, and E. Teller, J. Chem. Phys. 21, 1087 (1953).

${ }^{23}$ D. Vanderbilt, Phys. Rev. B 41, 7892 (1990).

${ }^{24}$ P. Giannozzi, S. Baroni, N. Bonini, M. Calandra, R. Car, C. Cavazzoni, D. Ceresoli, G. L. Chiarotti, M. Cococcioni, I. Dabo et al., J. Phys.: Condens. Matter 21, 395502 (2009).

${ }^{25}$ J. P. Perdew, K. Burke, and M. Ernzerhof, Phys. Rev. Lett. 77, 3865 (1996).

${ }^{26}$ H. Monkhorst and J. Pack, Phys. Rev. B 13, 5188 (1976).

${ }^{27}$ K. Chen, L. R. Zhao, J. Rodgers, and J. S. Tse, J. Phys. D 36, 2725 (2003). 
${ }^{28}$ R. M. Wentzcovitch, J. L. Martins, and G. D. Price, Phys. Rev. Lett. 70, 3947 (1993).

${ }^{29}$ J. F. Nye, Physical Properties of Crystals: Their Representation by Tensors and Matrices (Oxford University Press, USA, 1985).

${ }^{30}$ L. Vitos, Computational Quantum Mechanics for Materials Engineers: The EMTO Method and Applications (Engineering Materials and Processes) (Springer, Berlin, 2010).
${ }^{31}$ M. Moakher and A. N. Norris, J. Elast. 85, 215 (2006).

${ }^{32}$ J. T. Browaeys and S. Chevrot, Geophys. J. Int. 159, 667 (2004).

${ }^{33}$ See Supplemental Material at http://link.aps.org/supplemental/ 10.1103/PhysRevB.85.144112 for the explicit expression of the projector in case of hexagonal point group symmetry and for a detailed structural comparison of the used supercells. 